Claremont Colleges

Scholarship@ Claremont

All HMC Faculty Publications and Research

HMC Faculty Scholarship

8-1-2012

\title{
How the Cucumber Tendril Coils and Overwinds
}

Sharon J. Gerbode

Harvey Mudd College

Joshua R. Puzey

Harvard University

Andrew G. McCormick

Harvard University

L. Mahadevan

Harvard University

\section{Recommended Citation}

S. J. Gerbode, J. R. Puzey, A. G. McCormick, L. Mahadevan "How the cucumber tendril coils and overwinds" Science 337, 1087 (2012).

This Article - postprint is brought to you for free and open access by the HMC Faculty Scholarship at Scholarship @ Claremont. It has been accepted for inclusion in All HMC Faculty Publications and Research by an authorized administrator of Scholarship @ Claremont. For more information, please contact scholarship@cuc.claremont.edu. 
This copy is for your personal, non-commercial use only.

If you wish to distribute this article to others, you can order high-quality copies for your colleagues, clients, or customers by clicking here.

Permission to republish or repurpose articles or portions of articles can be obtained by following the guidelines here.

The following resources related to this article are available online at www.sciencemag.org (this information is current as of December 6, 2012 ):

Updated information and services, including high-resolution figures, can be found in the online version of this article at:

http://www.sciencemag.org/content/337/6098/1087.full.html

Supporting Online Material can be found at:

http://www.sciencemag.org/content/suppl/2012/08/29/337.6098.1087.DC1.html

This article cites $\mathbf{2 1}$ articles, 5 of which can be accessed free:

http://www.sciencemag.org/content/337/6098/1087.full.html\#ref-list-1

This article appears in the following subject collections:

Botany

http://www.sciencemag.org/cgi/collection/botany 
lack saprotrophic capability (1), $\mathrm{CO}_{2}$ enhancement of AMF for $\mathrm{N}$ scavenging likely increases decomposition by stimulating (i.e., priming) saprotrophs in soil through three potential mechanisms. First, AMF likely grow preferentially toward (15), and thus facilitate saprotrophs' access to, new organic patches (24). Second, AMF slowly release labile $\mathrm{C}$ for saprotrophs at relatively low concentrations (3), likely engendering a larger priming effect on decomposition than roots (fig. S7) (25-27). And third, rapid removal of newly released $\mathrm{NH}_{4}{ }^{+}$by AMF likely releases saprotrophs from metabolic repression (28).

Our findings indicate that $\mathrm{CO}_{2}$ enhancement of AMF may alter terrestrial ecosystem $\mathrm{C}$ dynamics by stimulating decomposition of soil organic $\mathrm{C}$ in AMF-active zones. This effect will likely occur in its interplay with other controlling factors such as temperature and plant species composition (29). In many agro- or grassland ecosystems where AMF dominate (1), but no aboveground $\mathrm{C}$ pool with an annual incremental increase exists, $\mathrm{CO}_{2}$ stimulation of AMF and organic $\mathrm{C}$ decomposition will mainly facilitate $\mathrm{C}$ turnover belowground, rather than ecosystem $\mathrm{C}$ sequestration (30). Even in forests with abundant AMF (e.g., tropical forests) (1), $\mathrm{eCO}_{2}$ stimulation of AMF, although creating a transient $\mathrm{C}$ sink in plant biomass by facilitating $\mathrm{N}$ transfer from soil to plants and partially alleviating $\mathrm{N}$ limitation on plants (31), is likely to reduce the largest carbon stocks (soil C) in the system. Also, our results suggest that the form, rather than just the total amount, of soil $\mathrm{N}$ might play a major role in mediating belowground $\mathrm{C}$ turnover and plant $\mathrm{N}$ acquisition under $\mathrm{eCO}_{2}$, thus offering a theoretical foundation for management of microbial $\mathrm{N}$ transformations in soil and plant $\mathrm{N}$ utilization to facilitate ecosystem $\mathrm{C}$ sequestration under future $\mathrm{CO}_{2}$ scenarios.

\section{References and Notes}

1. S. E. Smith, D. J. Read, Mycorrhizal Symbiosis (Academic Press, San Diego, ed. 2, 2008)

2. E. T. Kiers et al., Science 333, 880 (2011).

3. B. Drigo et al., Proc. Natl. Acad. Sci. U.S.A. 107, 10938 (2010).

4. I. Jakobsen, L. Rosendahl, New Phytol. 115, 77 (1990).

5. G. W. T. Wilson, C. W. Rice, M. C. Rillig, A. Springer, D. C. Hartnett, Ecol. Lett. 12, 452 (2009).

6. J. M. Tisdall, S. E. Smith, P. Rengasamy, Aust. J. Soil Res. 35, 55 (1997).

7. I. R. Sanders, R. Streitwolf-Engel, M. G. A. van der Heijden, T. Boller, A. Wiemken, Oecologia 117, 496 (1998).

8. K. K. Treseder, M. F. Allen, New Phytol. 147, 189 (2000).

9. O. Alberton, T. W. Kuyper, A. Gorissen, New Phytol. 167, 859 (2005).

10. M. C. Rillig, S. F. Wright, M. F. Allen, C. B. Field, Nature 400, 628 (1999).

11. S. Hu, F. S. Chapin III, M. K. Firestone, C. B. Field, N. R. Chiariello, Nature 409, 188 (2001).

12. K. H. Orwin, M. U. F. Kirschbaum, M. G. St John, I. A. Dickie, Ecol. Lett. 14, 493 (2011).

13. A. Hodge, A. H. Fitter, Proc. Natl. Acad. Sci. U.S.A. 107, 13754 (2010)

14. C. Tu et al., Glob. Change Biol. 12, 793 (2006).

15. A. Hodge, C. D. Campbell, A. H. Fitter, Nature 413, 297 (2001).

16. See supplementary materials on Science Online.

17. S. Solomon et al., Climate Change 2007: The Physical Science Basis Contribution of Working Group I to the Fourth Assessment Report of the Intergovernmental Panel on Climate Change (Cambridge Univ. Press, Cambridge, 2007).

18. L. Cheng et al., PLOS ONE 6, e21377 (2011).

19. A. J. Bloom, M. Burger, ]. S. R. Asensio, A. B. Cousins, Science 328, 899 (2010).

20. A. J. Bloom, D. R. Smart, D. T. Nguyen, P. S. Searles, Proc. Natl. Acad. Sci. U.S.A. 99, 1730 (2002)

21. H. J. Di et al., Nat. Geosci. 2, 621 (2009).
22. C. R. Fellbaum et al., Proc. Natl. Acad. Sci. U.S.A. 109, 2666 (2012).

23. M. Govindarajulu et al., Nature 435, 819 (2005).

24. P. Bonfante, I.-A. Anca, Annu. Rev. Microbiol. 63, 363 (2009).

25. R. P. Phillips, A. C. Finzi, E. S. Bernhardt, Ecol. Lett. 14, 187 (2011)

26. K. M. Carney, B. A. Hungate, B. G. Drake, J. P. Megonigal, Proc. Natl. Acad. Sci. U.S.A. 104, 4990 (2007).

27. M.-A. de Graaff, A. T. Classen, H. F. Castro, C. W. Schadt, New Phytol. 188, 1055 (2010).

28. D. Geisseler, W. R. Horwath, R. G. Joergensen, B. Ludwig, Soil Biol. Biochem. 42, 2058 (2010).

29. E. A. Davidson, I. A. Janssens, Nature 440, 165 (2006).

30. K. J. van Groenigen, C. W. Osenberg, B. A. Hungate, Nature 475, 214 (2011)

31. P. B. Reich et al., Nature 440, 922 (2006).

Acknowledgments: We thank F. Chapin III, D. Coleman, Y. Luo, R. Miller, and M. Rillig for valuable comments; M. Gumpertz for advice on statistical analyses; ]. Barton, W. Pursley, and E. Silva for technical assistance; and D. Watson and J. Morton for providing mycorrhizal inoculum. L.C. was primarily supported by a fellowship from U.S. Department of Agriculture (USDA)-Agricultural Research Service Plant Science Research Unit (Raleigh, NC) and in part by a USDA grant to S.H. (2009-35101-05351). S.H., L.C. and C.T. conceived experiments 1 to 4. K.O.B. and F.L.B. designed and maintained the long-term $\mathrm{CO}_{2}$ and $\mathrm{O}_{3}$ study. H.D.S and T.W.R. contributed to design of experiments 1 and 2. L.C. performed experiments 1 to 3 and the meta-analysis study; and C.T., F.L.B., and L.Z. performed experiment 4. L.C. and S.H. analyzed the data and mainly wrote the manuscript with inputs from all coauthors. The data reported in this paper are deposited in the Dryad Repository (http://dx.doi.org/10.5061/ dryad.b7f53).

Supplementary Materials

www.sciencemag.org/cgi/content/full/337/6098/1084/DC1

Materials and Methods

Figs. $S 1$ to $S 7$

Tables $\mathrm{S} 1$ and $\mathrm{S} 2$

References (32-79)

4 May 2012; accepted 10 July 2012

10.1126/science.1224304

\section{How the Cucumber Tendril Coils and Overwinds}

\section{Sharon J. Gerbode, ${ }^{1,2,3 *}$ Joshua R. Puzey, ${ }^{4 *}$ Andrew G. McCormick, ${ }^{5}$ L. Mahadevan ${ }^{1,2,4,5} \dagger$}

The helical coiling of plant tendrils has fascinated scientists for centuries, yet the underlying mechanism remains elusive. Moreover, despite Darwin's widely accepted interpretation of coiled tendrils as soft springs, their mechanical behavior remains unknown. Our experiments on cucumber tendrils demonstrate that tendril coiling occurs via asymmetric contraction of an internal fiber ribbon of specialized cells. Under tension, both extracted fiber ribbons and old tendrils exhibit twistless overwinding rather than unwinding, with an initially soft response followed by strong strain-stiffening at large extensions. We explain this behavior using physical models of prestrained rubber strips, geometric arguments, and mathematical models of elastic filaments. Collectively, our study illuminates the origin of tendril coiling, quantifies Darwin's original proposal, and suggests designs for biomimetic twistless springs with tunable mechanical responses.

$\mathrm{T}$ he transformation of a straight plant tendril into a helically coiled shape has inspired numerous studies since the 1800 s $(1-8)$, both from mechanistic and functional perspectives. Tendrils serve climbing plants by providing a parasitic alternative to building independently stable structural supports, allowing the plant to wend its way to sunlight and numerous ecological niches (9). During climbing, an initially straight tendril first finds and attaches to a support (fig. S1 and movie S1). Once tethered, the tendril coils by forming two oppositely handed helices connected by a "perversion" (Fig. 1, A and B), which was recognized by Darwin as a topological necessity given the clamped boundary conditions at each end of the tendril (3). This helical coiling axially shortens the tendril, hoisting the plant toward the attachment point (fig. S1 and movie S1).

Despite the long history of studying tendrils, the basic mechanism of tendril coiling has remained elusive. Historically, experimental studies of diverse tissues [reaction wood (10), hypocotyls (11), twining stems $(12,13)$, and leaves (14)] have addressed aspects of curvature generation, whereas

${ }^{1}$ School of Engineering and Applied Sciences, Harvard University, Cambridge, MA 02138, USA. ${ }^{2}$ Wyss Institute for Biologically Inspired Engineering, Harvard University, Cambridge, MA 02138, USA. ${ }^{3}$ Department of Physics, Harvey Mudd College, Claremont, CA 91711, USA. ${ }^{4}$ Department of Organismic and Evolutionary Biology, Harvard University, Cambridge, MA 02138, USA. ${ }^{5}$ Department of Physics, Harvard University, Cambridge, MA 02138, USA.

*These authors contributed equally to this work. †To whom correspondence should be addressed. E-mail: Im@seas.harvard.edu. 
theoretical treatments have incorporated intrinsic curvature or differential growth without addressing its origin or mechanical consequences $(6,15,16)$. Recent studies of tendril anatomy $(17,18)$ have provided a new twist by revealing an interior layer of specialized cells similar to the stiff, lignified gelatinous fiber (g-fiber) cells found in reaction wood (19). These cells provide structural support in reaction wood via tissue morphosis driven by cell-wall lignification, water flux, and oriented stiff cellulose microfibrils. The presence of a similar ribbon-like strip of g-fiber cells in tendrils suggests that the coiling of the soft tendril tissue may be driven by the shaping of this stiff, internal "fiber ribbon" (18).

We investigated the role of the fiber ribbon during tendril coiling in both Cucumis sativus (cucumber) and Echinocystis lobata (wild cucumber) (20). The g-fiber cells, identified in wild cucumber by using xylan antibodies in (18), are easily distinguished as a band of morphologically differentiated cells consistently positioned along the inner side of the helical tendril that lignify during coiling $(17,18)$. In straight tendrils that have not yet attached to a support (Fig. 1A), a faint band of immature g-fiber cells is barely visible by using darkfield microscopy (Fig. 1B), with no ultraviolet (UV) illumination signature, indicating the absence of lignification (Fig. 1C). In coiled tendrils (Fig. 1D), g-fiber cells are clearly visible (Fig. 1E) and lignified (Fig. 1F). The fiber ribbon consists of two cell layers, with the ventral layer on the inside of the helix showing increased lignification relative to the dorsal outer layer (Fig. 1, $\mathrm{G}$ and $\mathrm{H}$ ), which is consistent with earlier observations of increased lignification on the stimulated side of the tendril $(17,18)$. When a fiber ribbon is extracted from the coiled tendril by using fungal carbohydrolases [Driselase (Sigma-Aldrich, St. Louis, MO)] to break down the nonlignified epidermal tendril tissue (20), it retains the helical morphology of a coiled tendril, and furthermore, lengthwise cuts do not change its shape (Fig. 1I and fig. S2).

These observations suggest that tendril coiling occurs via asymmetric contraction of the fiber ribbon; the ventral side shrinks longitudinally relative to the dorsal side, giving the fiber ribbon its intrinsic curvature. The asymmetric contraction may be generated by a variety of dorsiventral asymmetries, including the observed differential lignification (Fig. 1H), variations in cellulose microfibril orientation as in reaction wood, or differential water affinities. For example, because lignin is hydrophobic the ventral cells may expel more water during lignification, driving increased cell contraction. This would be consistent with observations of extracted fiber ribbons that passively shrink and coil even further when dried but regain their original shape when rehydrated (movie S2). Dehydrated tendrils also exhibit this behavior because they are dominated by the stiff fiber ribbon (movie S3). Together, these facts suggest that the biophysical mechanism for tendril coiling is provided by the asymmetric contraction of the stiff fiber ribbon, whose resulting curvature is imposed on the surrounding soft tendril tissue. The perversions in a doubly supported tendril follow naturally from the topological constraint imposed by the prevention of twist at its ends.

To better understand the origin of curvature in fiber ribbons, we reconstituted the underlying mechanism using a physical model composed of two bonded, differentially prestrained silicone rubber sheets, similar to rubber models for shaping sheets (21-23). The first silicone sheet was uniaxially stretched, and an equally thick layer of silicone sealant was spread onto the stretched sheet. After the sealant was fully cured, thin strips were cut along the prestrained direction, yielding bilayer ribbons (Fig. 2A) with intrinsic curvature set by the relative prestrain, thickness, and stiffness of the two layers (fig. S3) (20). Like fiber ribbons, the initially straight physical models spontaneously form coiled configurations with two opposite-handed helices connected by a helical perversion (Fig. 2A, left).

However, there is an unexpected difference in mechanical behavior between the physical models and tendril fiber ribbons. When clamped at both ends and pulled axially, the physical model simply unwinds to its original uncoiled state (Fig. $2 \mathrm{~A}$ and movie S4). In contrast, in fiber ribbons we observed a counterintuitive "overwinding" behavior in which the ribbon coils even further
A

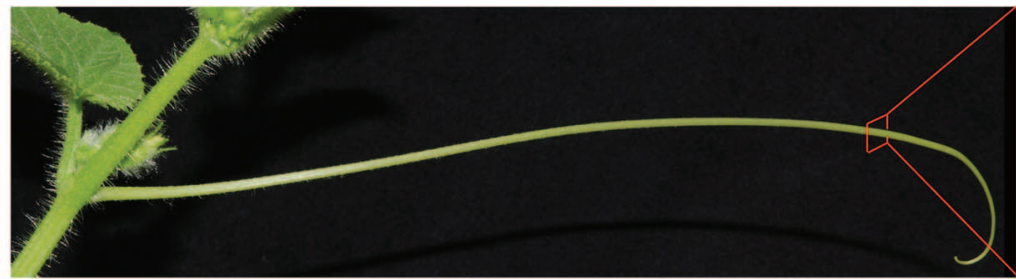

D

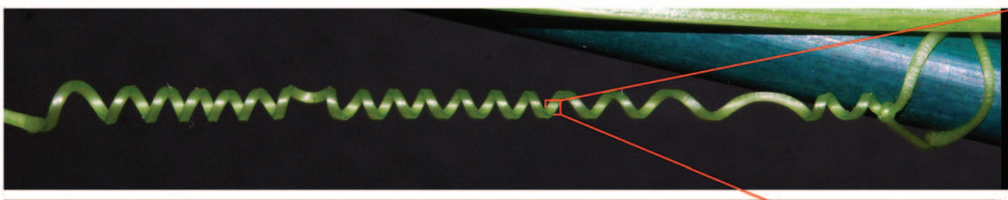

I

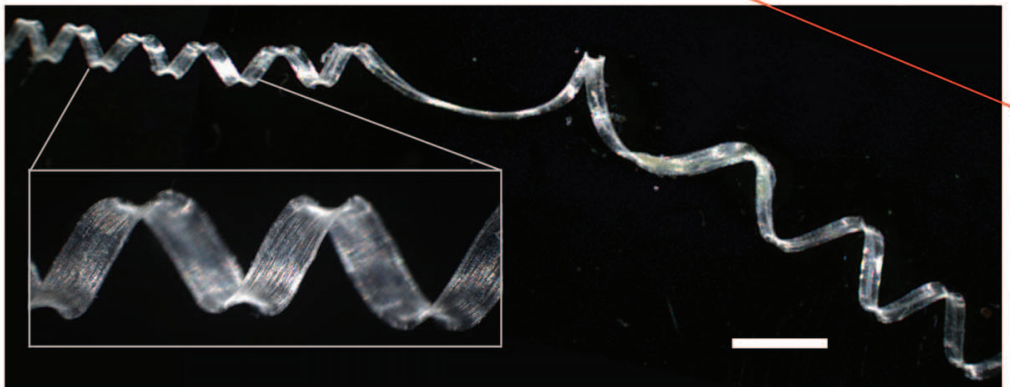

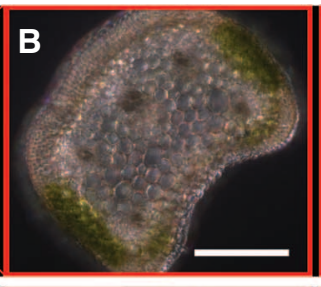
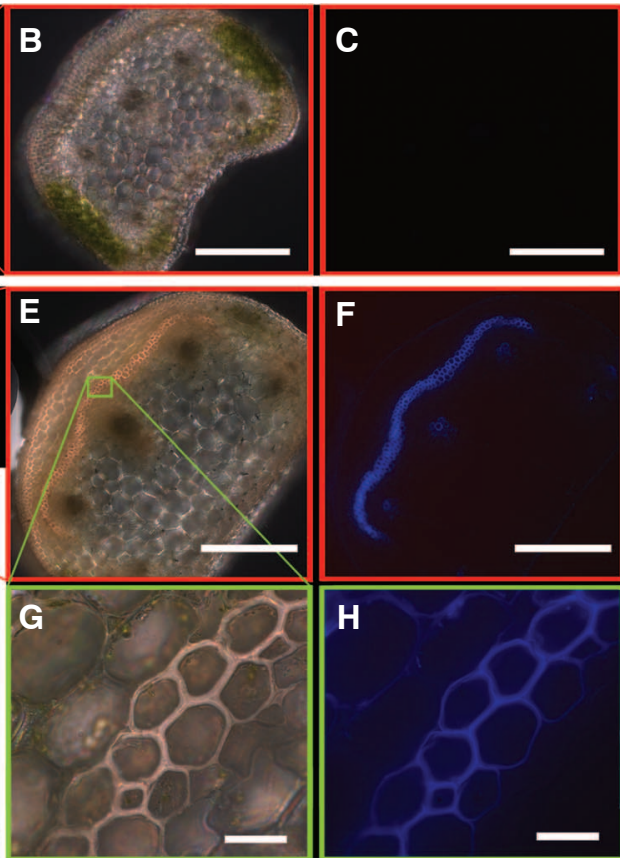

Fig. 1. Tendril coiling via asymmetric contraction. During coiling, a strip of specialized structural gelatinous fiber cells (the fiber ribbon) becomes lignified and contracts asymmetrically and longitudinally. (A to C) A straight tendril that has never coiled (A) lacks lignified g-fiber cells. In the tendril cross section, darkfield (B) and UV autofluorescence (C) show no lignin signal. (D to H) In coiled tendrils (D), the fully developed fiber ribbon consists of $\sim 2$ layers of highly lignified cells extending along the length of the tendril. In the tendril cross section, darkfield (E) and UV autofluorescence (F) show strong lignification in the fiber ribbon. In $(\mathrm{G})$ and $(\mathrm{H})$, increased magnification reveals that ventral cells (top left) are more lignified than dorsal cells. (I) The extracted fiber ribbon retains the helical morphology of the coiled tendril. (Inset) Higher magnification shows the orientation of $g$-fiber cells along the fiber ribbon. Scale bars, (B) and (C) $0.5 \mathrm{~mm},(\mathrm{E})$ and (F) $100 \mu \mathrm{m},(\mathrm{G})$ and (H) $10 \mu \mathrm{m},(\mathrm{I})$ $1 \mathrm{~mm}$. 
when pulled, adding turns on both sides of the perversion (Fig. 2A, right, and movie S5). Eventually though, under high enough tension the fiber ribbon unwinds, returning to a flat, uncoiled state as expected (movie S5).
Inspired by our observations of asymmetric lignification in fiber ribbons, which suggest that the inner layer is less extensible, we added a relatively inextensible fabric ribbon to the inside of a coiled physical model. To mimic lignified cells that resist compression, we added an incompressible copper wire to the exterior of the helix. The internal fabric ribbon prevents elongation, whereas the external copper wire prevents contraction. Together, these modifications increase

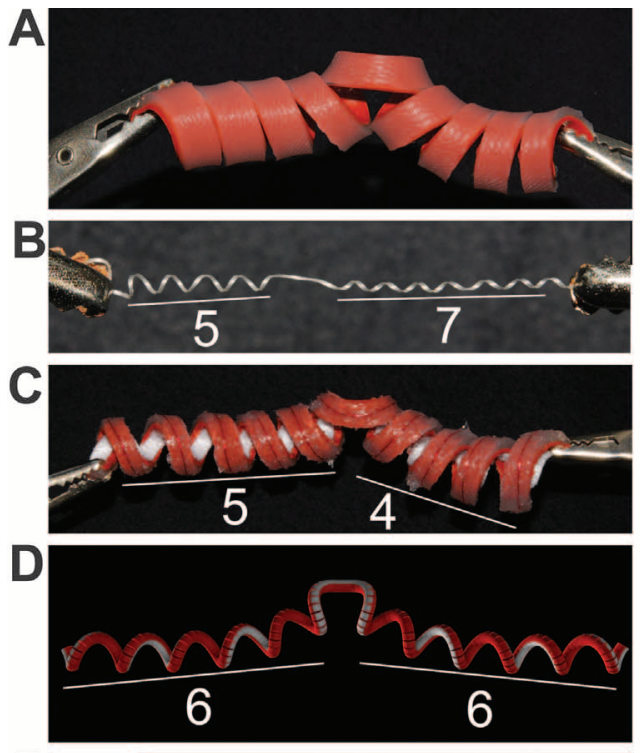

F

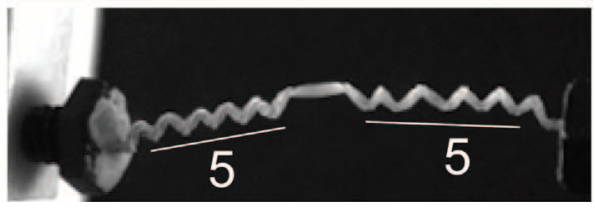

Fig. 2. Twistless springs unwinding and overwinding. (A) A silicone twistless spring with lower bending stiffness $B$ than twisting stiffness $C$ unwinds when pulled, returning to its original flat shape. (B) When a fiber ribbon is pulled, it initially overwinds, adding one extra turn to each side of the perversion (number of turns are indicated in white). (C) Overwinding is induced in the silicone model by adding a relatively inextensible (under tension) fabric ribbon to the interior of the helix and an inextensible (under compression) copper wire to the exterior.
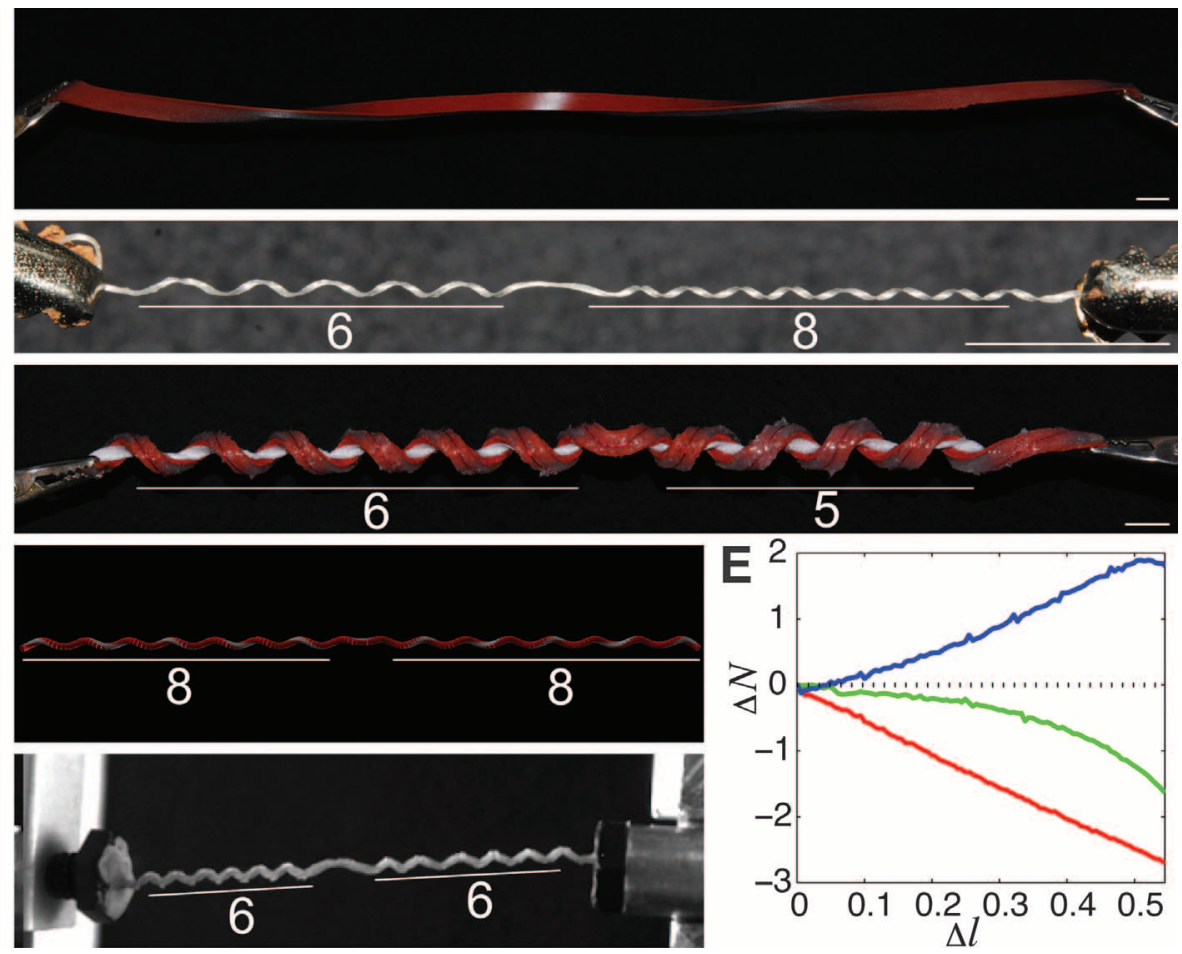

Together, these increase the ratio $B / C$. (D) When $B / C>1$, numerical simulations of elastic helical filaments recapitulate this overwinding behavior, which is consistent with physical and biological experiments. (E) Change in the number of turns in each helix $\Delta N$ is plotted versus scaled displacement $\Delta l$ for $B / C$ values $1 / 5$ (red), 1 (green), and 5 (blue). Overwinding becomes more pronounced with increasing $B / C$. (F) Overwinding is also observed in old tendrils, which have dried and flattened into a ribbon-like shape with $B / C>1$. Scale bars, $1 \mathrm{~cm}$.
Fig. 3. Mechanical consequences of overwinding. (A and B) Force extension curves for one young tendril that does not overwind (red curves) and one old tendril that exhibits substantial overwinding (blue curves). Each tendril was separated into a segment containing the helical perversion (dotted curves indicate perverted) and a segment with no perversion (solid curves indicate clamped). The dimensionless force $\tilde{F}$ is plotted against the scaled displacement $\Delta l$ (detailed definitions are available in the supplementary materials) in (A). The difference in scaled force due to the helical perversion $\Delta f=f$ (perverted) $-f$ (clamped) is plotted against $\Delta l$ in (B). The shaded range in (B) indicates variations in the fitted initial slope value. (C) Dimensionless forceextension curves are plotted for numerical filaments with $B / C$ values 1/5 (red), 1 (green), 5 (blue). (Inset) Log-linear plot of the same data. (D) The difference in force $\Delta \tilde{F}=$ $\tilde{F}$ (perverted) $-\tilde{F}$ (clamped) highlights the mechanical effect of the helical perversion. For $B<C$, the perversion always decreases the force needed to axially extend the filament; for $B>C$, the perversion initially decreases the force needed but eventually increases this necessary force at higher extensions. (Inset) $\Delta f$ is plotted against $\Delta l$ for direct comparison with the experimental data.
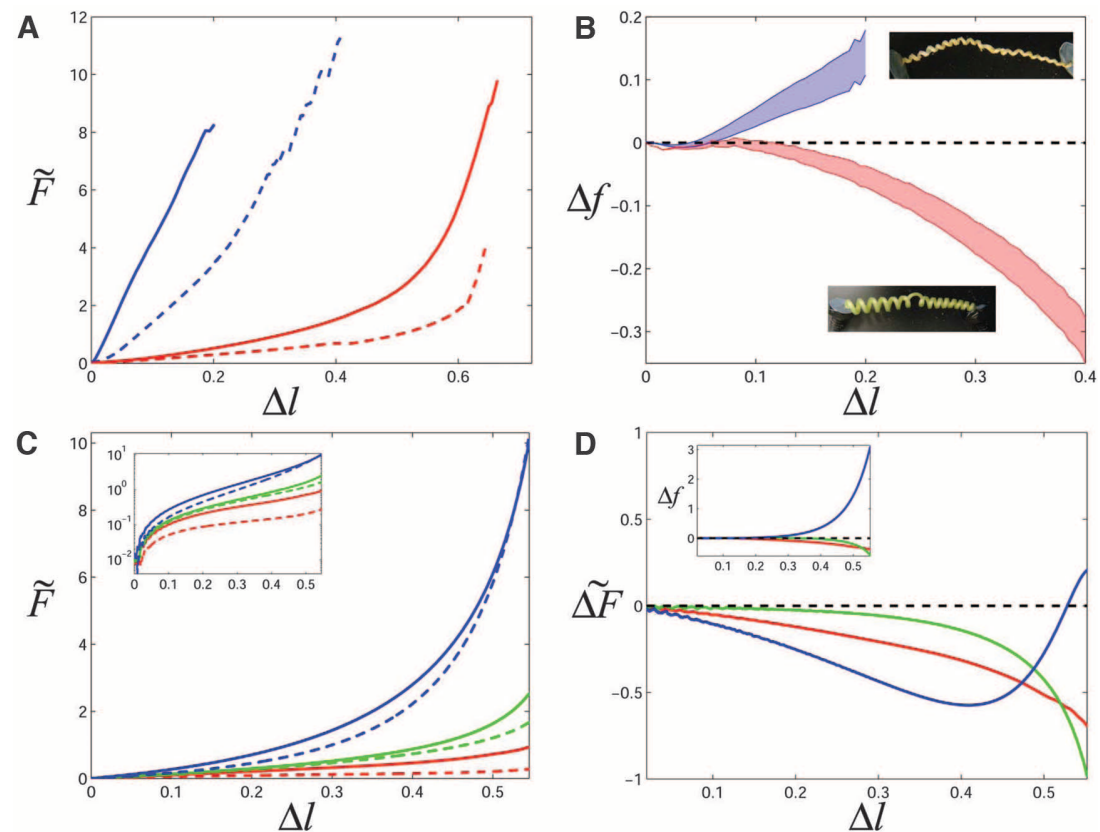
the model's effective bending stiffness relative to its twisting stiffness, fixing its intrinsic helix curvature while still allowing twist about its centerline (20). The modified model exhibited substantial overwinding (Fig. 2C and movie S6). Indeed, a single helix with infinite bending stiffness and fixed curvature cannot extend without its ends rotating. However, if one end may rotate, additional axial length can be accommodated by changing both pitch and radius to maintain constant curvature, resulting in additional helical turns (20). The perversion connecting helices of opposite handedness allows rotation and enables the addition of helical turns. By overwinding, each helix can thus geometrically accommodate axial extension without varying its curvature (fig. S4).

Of course, real tendril fiber ribbons have finite stretching and bending stiffness, and eventually at sufficiently high tensions, the helices unwind. To study overwinding in a fiber ribbon with finite bending and twisting stiffness, we modeled it mathematically as a filament composed of two equal-length, elastic helices of opposite handedness but identical intrinsic curvature $k_{0}$ and torsion $w_{0}$, and uniform bending stiffness $B$ and twisting stiffness $C$, connected by a single helical perversion (Fig. 2D, left). When the filament, initially at equilibrium, is pulled apart at its clamped ends, deviations from equilibrium values of curvature and twist lead to variations in the filament's total energy (20). Minimizing the energy of the extended filament numerically (20), we determined the filament shape and position as a function of the applied tension (Fig. $2 \mathrm{D}$, right). When $B / C<1$, the filament unwinds on extension, but when $B / C>1$, the filament overwinds (Fig. 2D and movies S7 and S8), and the number of additional turns $\Delta N$ increases with increasing $B / C$ (Fig. 2E) (24). For comparison, for a helical spring with a circular cross-section made of an isotropic material, $B / C=1+\mathrm{v}$, with Poisson ratio $v$ normally in the range $0<v \lesssim$ 0.5 so that typical springs exhibit minimal overwinding.

The observation of overwinding in fiber ribbons naturally leads to the question of whether entire tendrils also overwind. Whereas both young and old fiber ribbons always overwind, recently coiled, fully hydrated tendrils ("young" tendrils) do not overwind, but mature, dry tendrils ("old" tendrils) exhibit substantial overwinding (Fig. 2F and movies S9 and S10), and intermediate tendrils were variable in their overwinding behavior. The overwinding observed in old tendrils is likely due to the fact that as the tendril dries, the epidermal cells lose volume, and the tendril flattens down to a ribbon like shape similar to the internal fiber ribbon, so that $B / C>1$.

To investigate the mechanical and functional consequences of overwinding, we measured the force required to axially stretch tendrils using a custom force measurement setup (20). Forceextension curves measured for a total of 20 tendrils show a variety of mechanical responses; in
Fig. 3, we plot the dimensionless force $\tilde{F}$ against the scaled displacement $\Delta l$ (detailed definitions of $\Delta l$ and $\tilde{F}$ are in the supplementary text) for the two most extreme cases, a young tendril (red) and an old tendril (blue). For each, we show the results for a segment containing the perversion (Fig. 3; dotted curves indicate "perverted"), and another for a segment without it (Fig. 3; solid curves indicate "clamped"). In the young tendril, the perverted segment is always softer than the clamped segment (Fig. 3A). In contrast, the perverted segment of the old tendril is initially softer than the clamped segment but becomes stiffer at large extensions. Plotting the difference $\Delta f=$ $f$ (perverted) $-f$ (clamped), where the scaled force $f$ is obtained by dividing each force curve by its own initial slope (Fig. 3B), we see that for the young tendril in which no overwinding occurs, $\Delta f$ is always negative, indicating that the perversion consistently decreases the force necessary to stretch the tendril relative to the clamped case. However, in the old, overwinding tendril the perversion actually increases the force needed to stretch the tendril as $\Delta l$ increases.

To quantify the behaviors bounded by these two extreme tendril measurements, we also calculated force-extension curves using our mathematical models. The dimensionless force-extension curves for filaments with $B / C=1 / 5$ (red), 1 (green), and 5 (blue) are shown in Fig. 3C. Similar to the behavior of the young tendril, in the filament with $B / C=1 / 5$ (no overwinding), the presence of the perversion decreases the stiffness of the system - the force needed to axially extend the filament. However, the force response qualitatively changes when $B / C \gtrsim 3$, and the filament exhibits substantial overwinding. As in the old tendril, initially the perversion decreases the force needed to stretch the filament, but at large extensions, the perversion actually increases the force needed; the differential stiffness of the system is non-monotonic. Indeed, we observed that the difference $\Delta \tilde{F}=\tilde{F}$ (perverted) $-\tilde{F}$ (clamped) is always negative for filaments with $B<C$, whereas in overwinding filaments with large $B / C$ values, $\Delta \tilde{F}$ transitions to positive values at large extensions (Fig. 3D). Thus, in overwinding filaments a helical perversion initially softens the force response but eventually stiffens the filament relative to the clamped case, which is a behavior qualitatively different from earlier theoretical studies $(6,16)$, in which overwinding was not observed in the range of $B / C$ values studied. The difference in scaled force $\Delta f$ shown in the Fig. 3D inset is consistent with experimental observations (Fig. $3 \mathrm{~B}$ ), indicating that the unusual force-extension behavior shown in Fig. 3D explains the extremes observed in the two tendrils.

Collectively, our observations raise questions at an evolutionary level about the ubiquity of this mechanism in other tendril-bearing species and at a mechanical level about the functional principles of these soft twistless springs. Preliminary studies of Passiflora tendrils reveal a band of g-fibers, suggesting a similar coiling mechanism (fig. S5); however, both young and old coiled Passiflora tendrils exhibit overwinding (fig. S5 and movie S11). Although Cucurbitaceae and Passifloraceae are from the same phylogenetic clade, their tendrils have evolved independently (25), inviting future comparative studies between species as well as investigations of subcellular processes regulating asymmetric contraction. Functionally, the combination of mechanical asymmetry, helical perversions, and large ratios of bending to twisting stiffness creates an autoadaptive springy tendril, one that is initially soft because it can overwind and then stiffens strongly when deformed further. Darwin himself wrote that "the tendril strikes some object, and quickly curls round ... contracts into a spire, dragging up the stem, and forming an excellent spring" (3). Our study illuminates and quantifies this proposal biophysically while suggesting biomimetic variants of the humble helical spring.

\section{References and Notes}

1. A. Gray, Structural Botany: or Organography on the Basis of Morphology; To Which Is Added the Principles of Taxonomy and Phytography, and a Glossary of Botanical Terms (Ivison, Blakeman, Taylor, New York, 1880).

2. H. v. Mohl, Principles of the Anatomy and Physiology of the Vegetable Cell (John Van Voorst, London, 1852).

3. C. Darwin, On the Movements and Habits of Climbing Plants (John Murray, London, 1865).

4. R. Dastur, G. Kapadia, Ann. Bot. (London) 45, 279 (1931).

5. M. Jaffe, A. Galston, Annu. Rev. Plant Physiol. 19, 417 (1968).

6. A. Goriely, M. Tabor, Phys. Rev. Lett. 80, 1564 (1998)

7. H. Lisk, Bot. Gaz. 78, 85 (1924).

8. D. T. MacDougal, Annal. Bot. os-10, 373 (1896).

9. S. Isnard, W. K. Silk, Am. J. Bot. 96, 1205 (2009)

10. B. F. Wilson, R. R. Archer, Annu. Rev. Plant Physiol. 28, 23 (1977).

11. W. Silk, R. Erickson, Am. J. Bot. 65, 310 (1978).

12. J. L. Scher, N. M. Holbrook, W. K. Silk, Planta 213, 192 (2001)

13. S. Isnard, A. R. Cobb, N. M. Holbrook, M. Zwieniecki, J. Dumais, Proc. Biol. Sci. 276, 2643 (2009).

14. U. Nath, B. C. W. Crawford, R. Carpenter, E. Coen, Science 299, 1404 (2003).

15. J. B. Keller, Lect. Math Life Sci. 13, 257 (1980).

16. T. McMillen, A. Goriely, J. Nonlinear Sci. 12, 241 (2002).

17. C. G. Meloche, ]. P. Knox, K. C. Vaughn, Planta 225, 485 (2007).

18. A. J. Bowling, K. C. Vaughn, Am. J. Bot. 96, 719 (2009)

19. L. Goswami et al., Plant J. 56, 531 (2008).

20. Materials and methods are available as supplementary materials on Science Online.

21. Z. Chen, C. Majidi, D. J. Srolovitz, M. Haataja, Appl. Phys. Lett. 98, 011906 (2011).

22. S. Armon, E. Efrati, R. Kupferman, E. Sharon, Science 333, 1726 (2011)

23. T. Savin et al., Nature 476, 57 (2011)

24. A. Love, A Treatise on the Mathematical Theory of Elasticity (Courier Dover Publications, Mineola, NY, 1944).

25. P. F. Stevens, Angiosperm phylogeny Web site, www.mobot.org/MOBOT/Research/APweb/welcome.html (2008).

Acknowledgments: This research was supported by funding from the MacArthur Foundation, the Wyss Institute, 
and the Kavli Institute. S.].G., J.R.P., and L.M. designed the study. S.].G., J.R.P., A.G.M., and L.M. conducted the research. S.J.G. and J.R.P. performed the biological and biophysical experiments. S.J.G., A.G.M., and L.M. handled biophysical theory. S.J.G., J.R.P., A.G.M., and L.M. contributed analytical tools and reagents and analyzed data. S.J.G., J.R.P., and L.M. wrote the paper.
Harvard University has filed a patent application relating to a tunable, twistless overwinding spring based on the results of this study.

Supplementary Materials

www.sciencemag.org/cgi/content/full/337/6098/1087/DC1

Materials and Methods
Supplementary Tex

Figs. $\mathrm{S} 1$ to $\mathrm{S} 5$

References (26-29)

Movies S1 to S11

13 April 2012; accepted 19 June 2012

10.1126/science.1223304

\section{A Single Progenitor Population Switches Behavior to Maintain and Repair Esophageal Epithelium}

\author{
David P. Doupé, ${ }^{1,4 *}$ Maria P. Alcolea, ${ }^{1 *}$ Amit Roshan, ${ }^{1}$ Gen Zhang, $^{2}$ Allon M. Klein, ${ }^{2,3}$ \\ Benjamin D. Simons, ${ }^{2,4}$ Philip H. Jones $^{1} \dagger$
}

Diseases of the esophageal epithelium (EE), such as reflux esophagitis and cancer, are rising in incidence. Despite this, the cellular behaviors underlying EE homeostasis and repair remain controversial. Here, we show that in mice, EE is maintained by a single population of cells that divide stochastically to generate proliferating and differentiating daughters with equal probability. In response to challenge with all-trans retinoic acid (atRA), the balance of daughter cell fate is unaltered, but the rate of cell division increases. However, after wounding, cells reversibly switch to producing an excess of proliferating daughters until the wound has closed. Such fate-switching enables a single progenitor population to both maintain and repair tissue without the need for a "reserve" slow-cycling stem cell pool.
$\mathrm{M}$ urine esophageal epithelium (EE) consists of layers of keratinocytes. This tissue lacks structures such as crypts or glands that form stem cell niches in other epithelia (Fig. 1, A and B) (1-5). Proliferation is confined to cells in the basal layer (6). On commitment to terminal differentiation, basal cells exit the cell cycle and subsequently migrate to the tissue surface from which they are shed. Early studies suggested that all proliferating cells were functionally equivalent, but recent reports propose that a discrete population of slow-cycling stem cells is responsible for both maintenance and wound healing (7-11). This controversy and the importance of $\mathrm{EE}$ in disease motivated us to resolve the proliferative cell behavior in homeostatic $\mathrm{EE}$ and in tissue challenged by systemic treatment with the vitamin A metabolite all-trans retinoic acid (atRA) or acute local wounding $(12,13)$.

To investigate cell division rates in $\mathrm{EE}$, we used a transgenic label-retaining cell (LRC) assay (Fig. 1C) $(1,14,15)$. Doxycycline (DOX) induction of the fusion protein Histone-2B enhanced green fluorescent protein (HGFP) expression in

\footnotetext{
${ }^{1}$ Medical Research Council (MRC) Cancer Cell Unit, HutchisonMRC Research Centre, Cambridge CB2 OXZ, UK. ${ }^{2}$ Cavendish Laboratory, Department of Physics, ]. ]. Thomson Avenue, University of Cambridge, Cambridge CB3 OHE, UK. ${ }^{3}$ Department of Systems Biology, Harvard Medical School, 200 Longwood Avenue, Boston, MA 02115, USA. ${ }^{4}$ The Wellcome Trust/Cancer Research UK Gurdon Institute, University of Cambridge, Tennis Court Road, Cambridge CB2 1QN, UK.

*These authors contributed equally to this work. †To whom correspondence should be addressed. E-mail: phj20@cam.ac.uk
}

Rosa $26^{\mathrm{M} 2 \mathrm{rtTA}} /$ TetO-HGFP mice resulted in nuclear fluorescent labeling throughout the EE (Fig. 1D and fig. S1A). When DOX is withdrawn, HGFP is diluted by cell division, leaving $0.4 \%$ basal layer cells $(561$ out of 140,000$)$ retaining label after a 4-week chase (Fig. 1E and fig. S1B). Three-dimensional imaging showed that these LRCs had smaller nuclei than the surrounding keratinocytes and did not stain for the basal keratinocyte marker Keratin14 (0 out of 561 LRCs) (fig. S1, C and D). The stem cell markers CD34
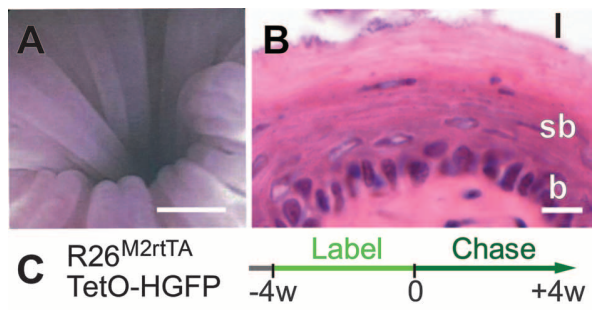

Fig. 1. Esophageal epithelium contains no slowcycling epithelial cells. (A) Microendoscopy showing esophageal lumen; scale bar, $\sim 500 \mu \mathrm{m}$. (B) Section of epithelium, basal layer (b), suprabasal layers (sb), and lumen (l); scale bar, $10 \mu \mathrm{m}$. (C) Protocol: Adult Rosa26 ${ }^{\text {M2rtA }} /$ /etO-HGFP mice treated with doxycycline (DOX) express HGFP (green). After DOX withdrawal, HGFP is diluted upon cell division, except in slow-cycling cells. (D and $\mathbf{E}$ ) Rendered confocal $z$ stacks, showing HGFP (green)

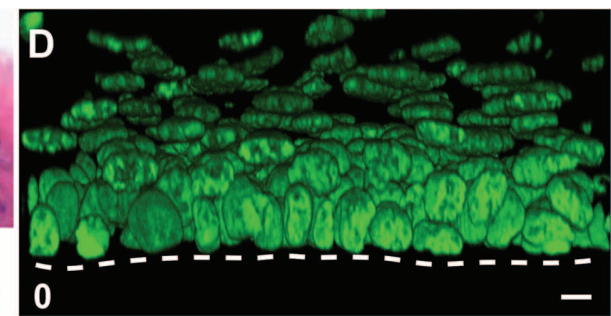

and $\operatorname{Lgr} 5$ were also undetectable in LRCs or other cells (figs. S2 and S3) $(2,4,10,16)$. However, $99.9 \%$ (2457 out of 2459) of LRCs were positive for the pan leukocyte marker CD45 (Fig. 1E, inset), comprising a mixture of Langerhans cells and lymphocytes (fig. S1, E and F). These findings lead to the unexpected conclusion that, unlike tissues such as the epidermis, there are no slowcycling or quiescent epithelial stem cells in $\mathrm{EE}$ $(1,17)$. Indeed, HGFP dilution in basal cells was strikingly homogeneous, suggesting that all cells divide at a similar rate of about twice per week (fig. S1G).

Although epithelial cells have the same rate of division, they may still differ in their ability to generate cycling and differentiated progeny. We therefore used inducible cre-lox-based genetic marking to investigate whether the proliferating cell population is heterogeneous and to quantify cell behavior $(18,19)$. The fate of single-cellderived clones was tracked in cohorts of adult Ahcre ${ }^{\mathrm{ERT}} \mathrm{R} 26^{\mathrm{flEYFP} / \mathrm{wt}}$ mice at multiple time points over a year after induction, during which period EE was homeostatic (Fig. 2A and fig. S4). Crucially, analysis of the composition of clones at 1 year showed that they were representative of unlabeled cells (fig. S5). Over the time course, clone number decreased through differentiation, whereas the size of the remaining clones progressively increased (Fig. 2, B and C). Although variation in labeling efficiency limits the accuracy with which the proportion of labeled cells can be estimated, within statistical error,

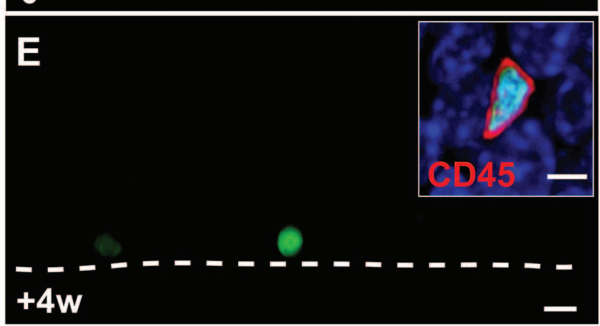

at time $0(D)$ and after 4-week chase (E). Scale bar, $10 \mu \mathrm{m}$. Dashed line indicates basement membrane. Inset shows CD45 (red) staining in HGFP-retaining cell at 4 weeks. 4',6-diamidino-2-phenylindole (DAPI), blue; scale bar, $5 \mu \mathrm{m}$. 